\title{
Route Selection for Vehicle Navigation and Control
}

\author{
Grantham Pang, Senior Member, IEEE and Ming-Hei Chu, Student Member, IEEE \\ Department of Electrical and Electronic Engineering \\ The University of Hong Kong \\ Email: gpang@,eee.hku.hk; mhchu@,eee.hku.hk
}

\begin{abstract}
This paper presents an application of neural-fuzzy methodology for the problem of route selection in a typical vehicle navigation and control system. The idea of the primary attributes of a route is discussed, and a neural-fuzzy system is developed to help a user to select a route out of the many possible routes from an origin to the destination. The user may not adopt the recommendation provided by the system and choose an alternate route. One novel feature of the system is that the neural-fuzzy system can adapt itself by changing the weights of the defined fuzzy rules through a training procedure. Two examples are given in this paper to illustrate how the route selection/ranking system can be made adaptive to the past choice or preference of the user.
\end{abstract}

\section{INTRODUCTION}

$\mathrm{W}$ ITH the advancement in sensor, communication and computer technologies nowadays, very current information on the road networks and street conditions (such as congestion) can be made available. A traffic control center has vast amount of information that can be distributed and utilized for vehicle navigation and control. This information can be transmitted through radio-wave and short-range beacons, or FM multiplex broadcasts. Most vehicle navigation systems nowadays already contain digital maps and detailed information of road networks and facilities, as well as sophisticated routing algorithms. Together with the up-to-the-minute information on the road conditions such as travel flow, congestion, road works and accidents, a navigation system can help to reduce the stress of driving by suggesting the best route to the destination. This paper presents an optimum route selection function for a vehicle navigation and control system. One novel feature of the proposed function developed based on neural-fuzzy methodology is that the system can be made adaptive to the user. After an origin-destination pair is specified, the driver can be presented with a set of feasible routes. A user-friendly system would provide a ranking of the routes based on the pre-defined model or behavior of the driver. This is an important feature because the driver is already overloaded with an abundant amount of information during driving. This ranking of feasible routes would turn the navigation system into a decision-support tool. Yet, depending on the mood or for any unknown reason, the driver may not always pick the route ranked number one on the list. The

Grantham Pang and Michael Ming-Hei Chu are with the Department of Electrical and Electronic Engineering, The University of Hong Kong, Pokfulam Road, Hong Kong. (e-mail: gpang@eee.hku.hk). system should be able to adapt itself based on the previous choice by the driver. This paper presents a neural-fuzzy training procedure for the route selection function to adapt itself to the driver.

\section{Route Selection Behavioral Studies}

There have been a number of studies on the behavior of drivers when faced with the issue of route selection. The fundamental point is that drivers are influenced by information from a variety sources. Yet, most existing vehicle navigation and control systems perform the route selection function by computing only the shortest time [1-3] or the shortest distance [4]. Although it is natural to assume that a driver would value travel time (which should be highly correlated with travel distance), studies [5] have shown that $6-14 \%$ of drivers would choose routes that have neither the shortest distance nor the shortest time.

In [6], the authors have pointed out there are many criteria in route selection: shortest distance, shortest time, most economical route etc.. Other criteria that could be used for deciding on an "optimum" route include the width of road, the pavement, the road type/surface and slope. A cost function and used in the optimum route searching algorithm, which is based on the $A^{*}$ heuristic search algorithm. One interesting point included in the algorithm is the number of turns in a route, which will increase the cost of the route.

Frank [7] has described the navigation assistance features of a navigation system. As a decision-support assistant, the route selection function should suggest an optimized route when a destination is specified. The route can be optimized for the shortest time, shortest distance, most use of freeways, fewest left turns at intersections.

Winsum [8] has approached the problem by recognizing that route choice is determined by a number of attributes of routes. Very often, the criteria for route selection are not independent. In his approach, a score, which is called aggregated value, is computed for each route, and the "best" route is obtained based on the score. His route selection algorithm is based on the calculation of the aggregated value, which is essentially a summation of the value of each route attribute, modified by a weight of the attribute. A major problem of this method would be the determination of the set of weights of the road attribute.

Suga et al. [9] suggested a method to find an optimum route based on the calculation of route-cost using the Dijkstra search algorithm. The route-cost is simply the 
addition of every link cost in every road-link passed from the starting point to the destination. However, a number of assumptions are made on the meaning of "optimum". In the paper, a route that takes the shortest time to reach the destination is defined as optimum. However, as discussed later in this paper, this point of view may not be the best in all situations. The same approach was used by Shimizu et al. [10] in their optimum route guidance algorithm.

To conclude, route selection can be viewed as a complex decision process that involves the consideration of many factors and the selection of one of the many alternatives [11]. The route attributes that influence the choice include travel time, cost, travel distance, the number of traffic signals, stop signs, right turns, scenery, roadside development etc.. This paper is an extension from $[12,13]$ in many ways. First, the number of rules developed and the format are different. The antecedents of a rule can now accommodate two or additional route attributes. In addition, the neural-fuzzy system is designed to adjust the rule weightings rather than the shape of the output membership functions. These extensions would allow for more features and flexibility to the design of the system.

\section{ROUTE PRIMARY ATTRIBUTES}

The primary attributes of a route is discussed in this paper. A route attribute is a characteristic of a route, which is used by a driver as an assessment criterion in route selection. As there is a wide variety of attributes describing each route, it is convenient to classify them.

The primary attributes are defined as travel distance, travel time, degree of congestion, travel cost/toll (use of highway) and degree of difficulty of a route. These five attributes are called the primary attributes because they are primary in the sense that they are the important attributes used by drivers in the assessment of a route.

All other attributes are then called secondary attributes. They may include:

- minimum number of right turn,

- total number of turns,

- familiar route,

- pavement or road type/surface,

- slope of the road,

- scenery,

- number of traffic signals, number of stop signs etc.

In fact, some of these can easily be embodied within the primary attribute "degree of difficulty of the road". This classification will ease the development of an intelligent route selection system. From now on, the discussion will be focused on the primary attributes of a route.

Given a set of origin-destination (O-D) pair, there could be many feasible routes for a driver. Each of these feasible routes has different scores/values in their primary attributes. One route may have a high score in one attribute (e.g. shortest distance) but a low score in another attribute (e.g. very congested route). During the decision-making process, a driver has to assess the various attribute scores of each feasible route, and perform a tradeoff when deciding on a route.

Each attribute is designed to have a score between zero and one. For an O-D pair, let there be $J$ number of feasible routes. Also, let the number of primary attributes be five as described above. The notation $x_{i}^{j}$ is used to describe the score of attribute $i$ of route $j$ where $i=1, \ldots, 5$ and $j=1, \ldots, \mathrm{J}$.

It should be noted that the some objective measures can be used on the attributes "degree of congestion" and "degree of difficulty". On congestion, for example, feasible route $k$ would be given a low score value when the route is not very congested, and a high value (tends to one) if congested. The score could be calculated by a formula that depends on the queue length and/or the estimated total waiting/idle time when traveling on that route.

As for the other three primary attributes (time, distance and toll/cost), the nature of the original route data is continuous. All the data must be massaged/treated before they can be used in the subsequent development of a neural-fuzzy system. With the minimum and maximum value in a set of values, the input data can be mapped/massaged to the range between 0 and 1 as follows:

\section{NEURAL-FUZZY APPROACH}

As discussed in section three, a driver may select a route based on many different factors. The primary

$$
\text { massaged value }=\frac{\text { actual value }- \text { minimum value }}{\text { maximum value }- \text { minimum value }}
$$

factors/attributes are: travel distance, travel time, degree of congestion, travel cost and degree of difficulty of travel. The optimum route search function (Figure 1) is a decision-making assistant to the driver in route selection.

For a real-time dynamic system, it is perceived that at a particular instance of time, a number of feasible routes which have different set of attributes can be considered by the driver. The driver has to make a decision based on the

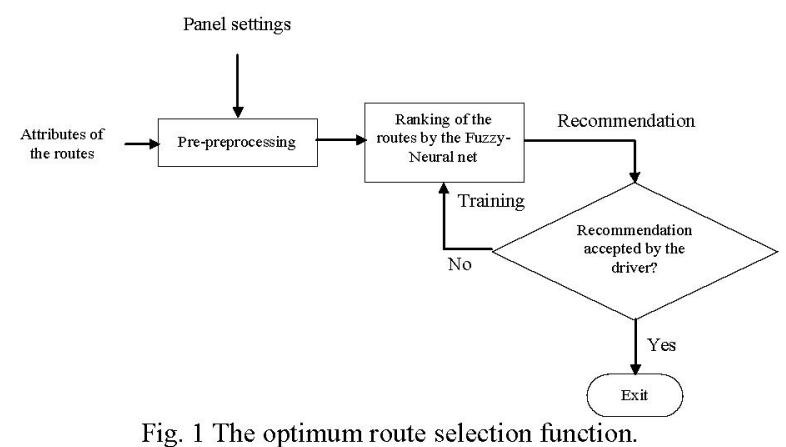

relative importance of the different factors for route selection. There could be some heuristics in route selection but some preferences could be difficult to express in 
words.

A fuzzy-neural approach [11] was developed for adaptive route selection for navigation systems. The architecture of the fuzzy-neural network is shown in Figure 2. The network is essentially a parallel implementation of a fuzzy system using a structured multi-layer neural network. The structure involves the construction of a fuzzification sub-network and a defuzzification sub-network. The two sub-networks are integrated in such a way that the structure and decision-making process of the original fuzzy system can be fully retrieved from its network implementation. The corresponding neural network should have the same performance as the original fuzzy system.

Other than the input and output layers, it has three hidden layers that represent membership functions and fuzzy rules. Referring to Figure 2, the second layer is the input membership functions or fuzzification layer. Neurons in this layer represent fuzzy sets used in the antecedents of a fuzzy rule. Layer 3 is the fuzzy rule layer. Each neuron is this layer corresponds to a single fuzzy rule. For example, neuron R2, which corresponds to Rule 2, receives inputs from neurons "short" (time) and "high" (toll). A fuzzy operator can be used to obtain a single number that represents the result of evaluating the two antecedents. The conjunction of them is carried out by the fuzzy operation "intersection", implemented by the "product" operator.

Rule 2:

IF time is SHORT and toll is HIGH, THEN score is FAIR.

The output from the neuron $\mathrm{R} 2$ represents the firing strength of Rule 2. The weights between Layer 3 and Layer 4 represent the normalized degrees of confidence of the corresponding fuzzy rules. For example, w2 is the weight denoting the certainty factor of Rule 2, connecting R2 with the neuron "fair" in the output membership layer (i.e. layer 4). Neurons in layer 4 represent fuzzy sets used in the consequent part of the fuzzy rules.

An output membership neuron would receive inputs from the corresponding fuzzy rule neurons and combines them by using the fuzzy operator union. This operation can be implemented by the probabilistic OR (i.e. the algebraic sum). Finally, layer $\mathbf{5}$ is the defuzzification layer. Each neuron is this layer represents a single output of the neural-fuzzy system. The sum-product composition method is used in this paper. It calculates the crisp output as the weighted average of the centroids of all output membership functions.

Hence, the neural-fuzzy system is essentially a multi-layer neural network implementation of a fuzzy system. Thus, the standard back-propagation algorithm can be used for training the system. The weights between layer 3 and 4 are adjusted during the training of the route selection system.

\section{SYSTEM IMPLEMENTATION AND RESULTS}

The objective is to illustrate how the navigation system can adapt to the preference of route selection by the user in the past. As an illustration, we have the following seven fuzzy rules:

Rule 1:

IF time is SHORT, THEN score is VERY GOOD.

Rule 2 :

If time is SHORT and toll is HIGH, THEN score is FAIR. Rule 3:

IF time is LONG and difficulty is LOW, THEN score is GOOD.

Rule 4:

If distance is LONG and congestion is LOW, THEN score is GOOD.

Rule 5 :

IF distance is SHORT and difficulty is HIGH, THEN score is BAD.

Rule 6:

IF distance is SHORT and congestion is HIGH, THEN score is VERY BAD.

Rule 7 :

IF toll is LOW, THEN score is VERY GOOD.

The input membership functions for SHORT and LOW are the same and shown in Fig. 3. The input membership functions for LONG and HIGH are the same and shown in Fig. 4. Figures 5-9 are the output membership functions for VERY BAD, BAD, FAIR, GOOD and VERY GOOD respectively.

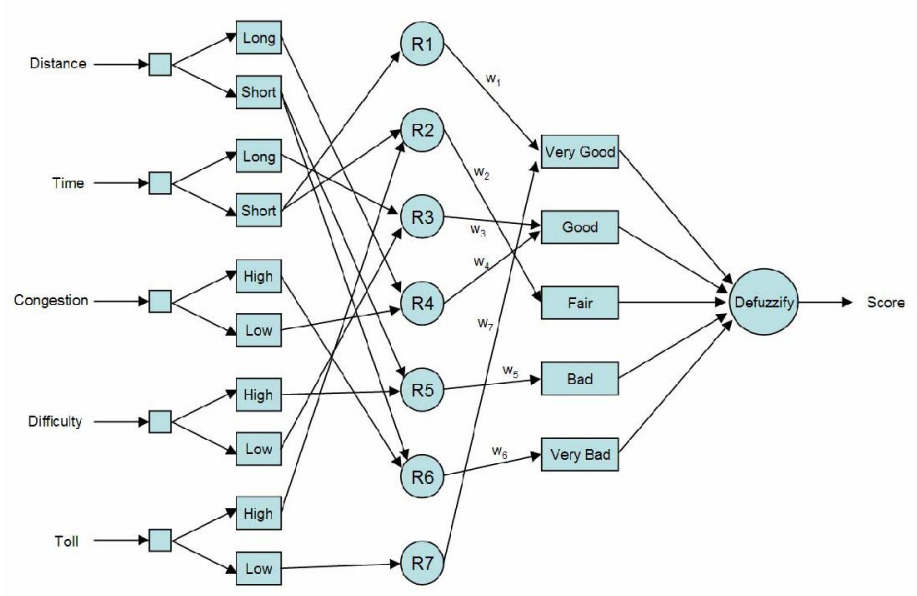

Fig. 2 The structure of the Fuzzy-Neural Network 


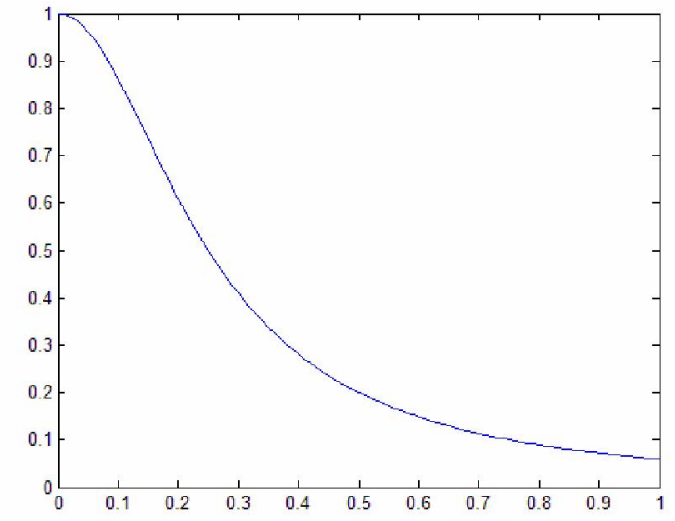

Fig. 3 The input membership function of "Distance is SHORT" "Time is SHORT", "Congestion is LOW", "Difficulty is LOW" and "Toll is LOW".

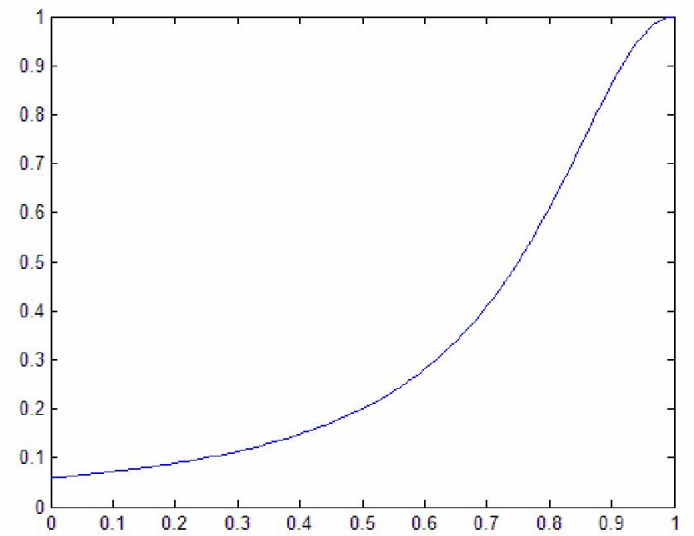

Fig. 4 The input membership function of "Distance is LONG", "Time is LONG", "Congestion is HIGH", "Difficulty is HIGH" and "Toll is HIGH".

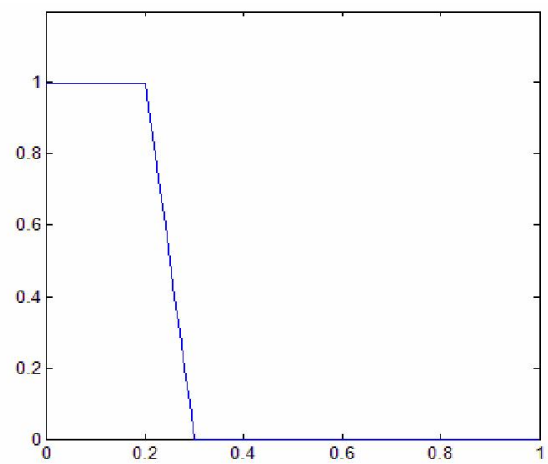

Fig. 5 The output membership function of "Very Bad".

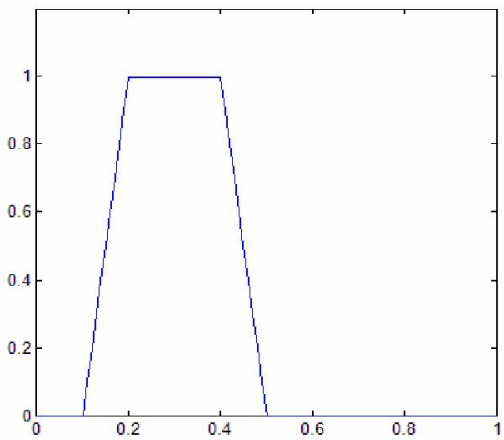

Fig. 6 The output membership function of "Bad".

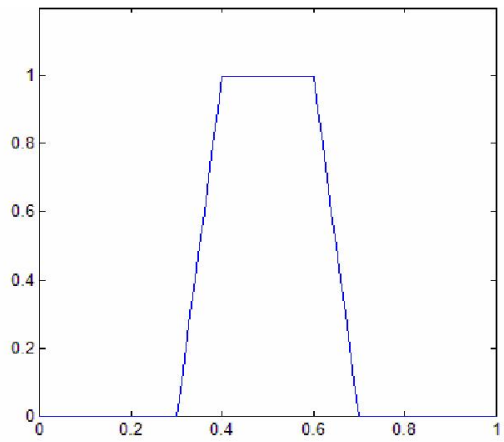

Fig. 7 The output membership function of "Fair".

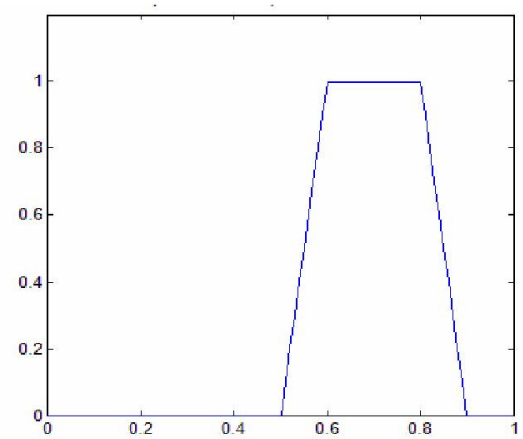

Fig. 8 The output membership function of "Good".

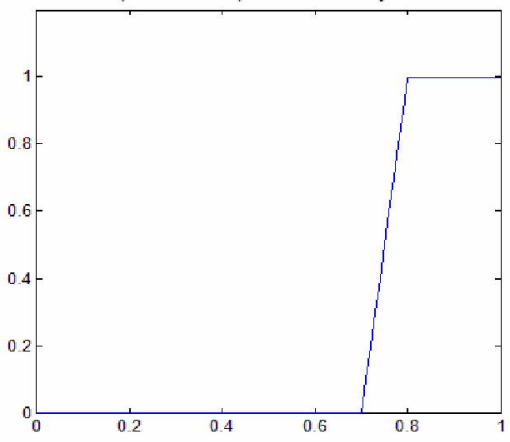

Fig. 9 The output membership function of "Very Good". 
TABLE I. PRIMARY ATTRIBUTE MATRIX OF ROUTE 1 AND ROUTE 2

\begin{tabular}{|l|c|c|c|c|c|}
\hline & Distance & Time & Congestion & Difficulty & Toll \\
\hline Route 1 & 0.60 & 0.40 & 0.70 & 0.50 & 0.30 \\
\hline Route 2 & 0.60 & 0.40 & 0.25 & 0.80 & 0.80 \\
\hline
\end{tabular}

A test example is shown which simulates the preference of the driver to avoid congestion. The testing procedures are given below:

1. The weights of all seven fuzzy rules are initially set to the value of 1 .

2. The Neural-Fuzzy (NF) Network is formed according to the fuzzy rules.

3. A score is obtained by running the NF net for each feasible route.

4. A ranking is obtained based on the scores.

\begin{tabular}{ll}
\hline Route $1=[0.60,0.40,0.70,0.50,0.30]$ & 0.6370 \\
Route $2=[0.60,0.40,0.25,0.80,0.80]$ & 0.6215 \\
\hline
\end{tabular}

Route 1 has the highest score, which is the recommended route.

5. Suppose the current user decides to choose Route 2 instead of Route 1 for the reason that Route 2 has a low congestion attribute $(0.25)$. The score of the chosen route is then exchanged with Route 1 and the following pair of training data is obtained:

\begin{tabular}{l}
{$[0.60,0.40,0.70,0.50,0.30] \longrightarrow 0.6215$} \\
{$[0.60,0.40,0.25,0.80,0.80] \longrightarrow 0.6370$} \\
\hline
\end{tabular}

6. During the training of the NF net, the weights of each fuzzy rule are changed. The weight changes of each fuzzy rule are shown in the following figure:

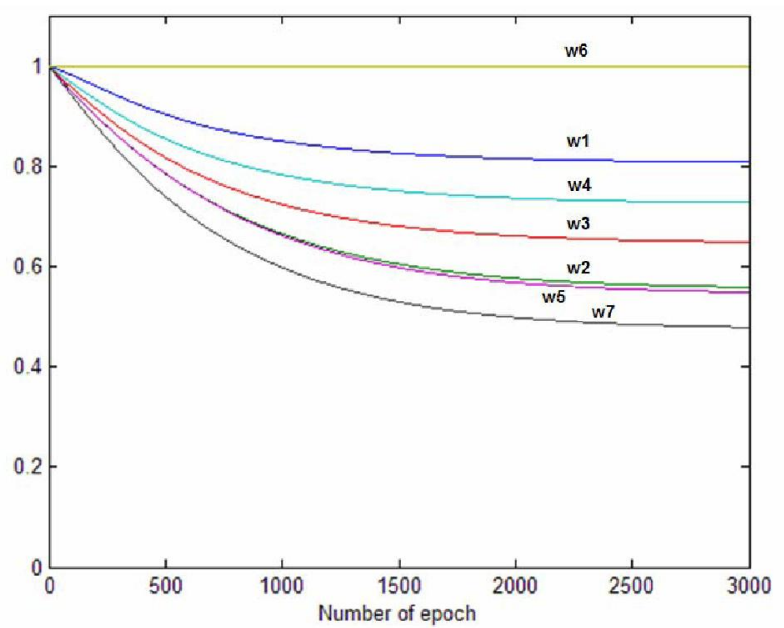

Fig. 10 Weights of each fuzzy rule after training (Example 1)

Some explanations on Example 1 are given below. It can been read from Rule 2 and Rule 7 that the original design of the fuzzy system favors low toll (low travel cost). Yet, the final choice of the user is Route 2, which has a higher attribute on toll/cost (the value is 0.80 ) but much less congested (the value is 0.25 instead of 0.70 of Route 1). Hence, the route selection system should learn this latest preference of the driver, and adapt itself to suit the user for future rankings.

The data for retraining the neural-fuzzy system can be obtained by interchanging the two score values. The argument is that if the same two routes are presented to the system for ranking in the next round, it is desired that the system would give a higher score for Route 2 than Route 1.

Indeed, from the weights of the seven fuzzy rules after training (initially, the weights are all ones), the weight of fuzzy rule 7 (IF toll is LOW, then SCORE is VERY GOOD) has dropped. This clearly reflects that the user is no longer in favor of low toll any more. On the other hand, rule 6 and rule 4 emphasize on low congestion, and their weights remain high even at the end of the training procedure.

Another test example is shown which simulates the preference of the driver to avoid long traveling time. Two feasible routes are given in Table II.

TABLE II. PRIMARY ATTRIBUTE MATRIX OF ROUTE 1 AND ROUTE 3

\begin{tabular}{|c|c|c|c|c|c|}
\hline & Distance & Time & Congestion & Difficulty & Toll \\
\hline Route 1 & 0.60 & 0.40 & 0.70 & 0.50 & 0.30 \\
\hline Route 3 & 0.50 & 0.30 & 0.80 & 0.60 & 0.80 \\
\hline
\end{tabular}

A ranking is obtained based on the scores from the NF net.

\begin{tabular}{ll}
\hline Route $1=[0.60,0.40,0.70,0.50,0.30]$ & 0.6370 \\
Route $3=[0.50,0.30,0.80,0.60,0.80]$ & 0.5584 \\
\hline
\end{tabular}

Route 1 has the highest score, which is the recommended route.

1. Suppose the current user would choose Route 3 that has the time attribute of 0.30 , the lowest among the routes. The score of the chosen route is then exchanged with Route 1 and the following pair of training data is obtained:

$[0.60,0.40,0.70,0.50,0.30] \longrightarrow 0.5584$
$[0.50,0.30,0.80,0.60,0.80] \longrightarrow 0.6370$

2. During the training of the NF net, the weights of each fuzzy rule are changed. The weight changes of each fuzzy rule are shown in the following figure: 


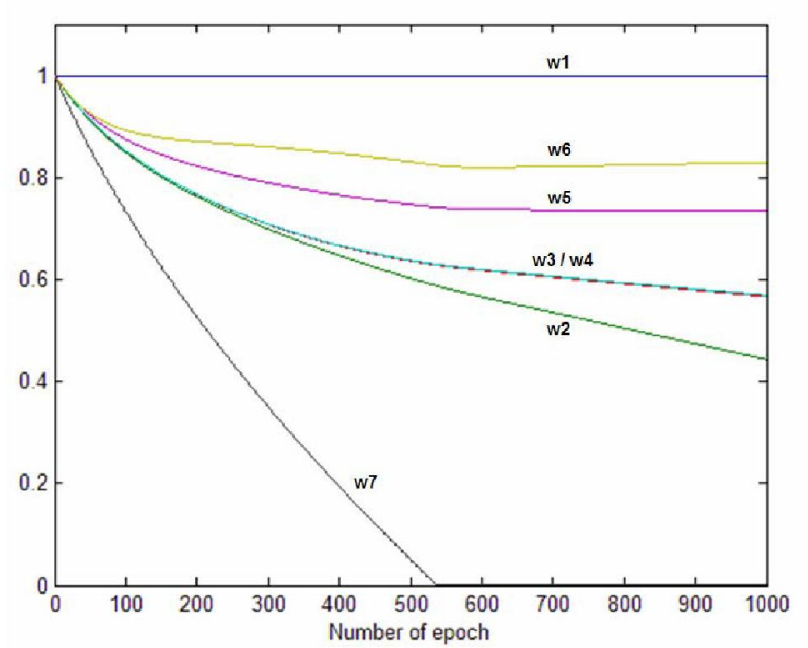

Fig. 11 Weights of each fuzzy rule after training (Example 2)

In this example, the weight for rule 7 becomes zero at the end of training. This indicates that the recent selection of the user is no longer in line with the original fuzzy rule 7. Another point to observe is that fuzzy rule 1 (IF time is SHORT, then SCORE is VERY GOOD) remains strong throughout, which indicates that the user indeed likes short travel time. The weights of rule 5 and rule 6 have dropped a bit to reflect that Route 3 is still acceptable even if the route is slightly more difficult and slightly more congested than Route 1.

\section{CONCLUSION}

An adaptive, neural-fuzzy based route selection function has been developed for a vehicle navigation and control system. The route recommendation/ranking is tailored for the driver and "optimal" to his/her own preference. This paves the way for an intelligent navigation system that can provide individualized travel support.

\section{REFERFNCES}

[1] N.B. Hounsell, M. McDonald and R.A. Lambert, "The integration of SCOOT and dynamic route guidance", Proc. Sixth Int. Conf. on Road Traffic Monitoring and Control, IEE, pp. 168-172, April 1992

[2] G. Hoffmann and J. Janko, "Travel times as a basic part of the LISB guidance strategy", Proc. Third Int. Conf. on Road Traffic Control, IEE, pp. 6-10, May 1990.

[3] T. van Vuren and D. van Vliet, Route Choice and Signal Control, Avebury ITS Institute for Transport Studies, Ashgate Publishing Ltd., 1992.

[4] T. Saito, J. Shima, H. Kanemitsu, Y. Tanaka, "Automobile navigation system using beacon information", Proc. 1st International Conf. on Vehicle Navigation \& Information Systems (VNIS), Toronto, Canada, pp. 139-144, 1989.

[5] G. Beccaria, A. Bolelli, "Modelling and assessment of dynamic route guidance : the Margot Project", Proc. 3rd International Conf. on Vehicle Navigation \& Information Systems (VNIS), Norway, pp. 117-124, Sept 1992.

[6] H. Wang, B. Zhang, "Route planning and navigation system for an autonomous land vehicle", Proc. 3rd International Conf. on Vehicle Navigation \&\& Information Systems (VNIS), Norway, pp. 135-140, Sept 1992

[7] D. Frank, "Information systems: an integral part of future vehicles", driver route information systems", Proc. 1st International Conf. on
Vehicle Navigation \& Information Systems (VNIS), Toronto, Canada, pp. 85-88, 1989.

8] W. van Winsum, "Selection of routes in route navigation system", in Driving Future Vehicles, A. M. Parkes and S. Franzen Ed., Taylor \& Francis, 1993, pp. 193-203.

[9] Y. Suga, Y. Nakamura and K. Owada, "Optimum and alternate route calculations for in-vehicle navigation system", Proc. of 2 nd World Congress on Intelligent Transport Systems, pp. 605-609, Nov. 9-11, Yokohama, 1995.

[10] H. Shimizu, M. Kobayashi and Y. Yonezawa, "An optimum route guidance algorithm of a traffic network", Proc. of 2nd World Congress on Intelligent Transport Systems, pp. 2045-2050, Nov. 9-11, Yokohama, 1995.

[11] P. P. Jovanis, R. Kitamura, "User perceptions and safety implications of in-vehicle navigation systems", Proc. 1st International Conf. on Vehicle Navigation \& Information Systems (VNIS), Toronto, Canada, pp. 113-116, 1989.

[12] Pang, G.K..H., Takahashi, K., Yokota, T. and Takenaga, H. "Adaptive route selection for dynamic route guidance system based on fuzzy-neural approaches", IEEE Transactions on Vehicular Technology, Vol. 48, No.6, pp. 2028-2041, November 1999.

[13] Pang, G. K. H., K. Takahashi, T. Yokota, H. Takenaga, "Intelligent Route Selection for In-vehicle Navigation System", Transportation Planning \& Technology , Vol. 25, Number 3, pp. 175-213, September 2002, ISSN/ISBN: 0308-1060. 